\title{
A Prospective Randomized Clinical Trial of Efficacy of Algorithm-Based Point of Care Guided Hemostatic Therapy in Cyanotic Congenital Heart Disease Surgical Patients
}

\author{
Ameya Karanjkar ${ }^{1}$ Poonam Malhotra Kapoor ${ }^{1}$ Sandeep Sharan ${ }^{1}$ Vandana Bhardwaj ${ }^{1}$ \\ Vishwas Malik $\quad$ Suruchi Hasija' $\quad$ Arindam Choudhury ${ }^{1}$ Ujjwal Kumar Chowdhury² ${ }^{2}$ P. Rajashekar ${ }^{2}$ \\ 1Department of Cardiac Anaesthesia, Cardiothoracic Centre, All \\ India Institute of Medical Sciences, New Delhi, India \\ 2Department of Cardiothoracic and Vascular Surgery, \\ Cardiothoracic Centre, All India Institute of Medical Sciences, \\ New Delhi, India \\ Address for correspondence Poonam Malhotra Kapoor, MD, DNB, \\ MNAMS, FIACTA (Hony), FTEE (Hony), FISCU (Hony), Department \\ of Cardiac Anaesthesia,7th floor, Cardiothoracic Centre, All India \\ Institute of Medical Sciences, Ansari Nagar, New Delhi, 110029, \\ India (e-mail:drpoonamaiims@gmail.com).
}

J Card Crit Care TSS 2020;3:8-16

Abstract

Keywords
- blood component
therapy
- TACO
- TRALI
- ARDS
- conventional tests
- cyanotic heart surgery
- POC algorithm
- point of care (POC)
- ROTEM
- sonoclot

Objective Point of care (POC) testing-based algorithm-guided hemostatic therapy has been used in adult as well as pediatric cardiac surgical patients to administer blood components. The authors hypothesized that implementation of POC-based algorithm in pediatric cyanotic congenital surgical patients would reduce the exposure to blood component therapy and improve the clinical outcome.

Design Prospective randomized control trial.

Setting Single-center academic institute.

Participants One hundred seventy pediatric congenital cyanotic surgical patients. Intervention Implementation of $\mathrm{POC}$ and conventional tests-based algorithms.

Measurements and Main Results Algorithm-based blood component therapy was administered in each group. There were no group differences regarding the demographic, clinical, and laboratory characteristics. Amount of packed red blood cells (PRBCs), fresh frozen plasma (FFP), platelets, and cryoprecipitate (primary outcomes) administered was significantly lower in POC group than that in the conventional group $(p<0.001)$. Among the secondary outcomes, the chest drain output at 6,12 , and 24 hours and number of re-explorations was comparable among both the groups. The duration of mechanical ventilation, duration of intensive care unit (ICU) stay, and hospital stay was significantly short in POC group $(p=0.008,<0.001$ and 0.019 , respectively).

Conclusion Implementation of POC-based algorithm-guided hemostatic therapy reduced the exposure to blood and blood component therapy and was associated with reduced ICU and hospital stay in pediatric congenital cyanotic surgical patients.

\section{Introduction}

Surgery for congenital cyanotic heart disease is associated with an increased tendency to bleed. ${ }^{1}$ There are various causes for bleeding tendency which include erythrocytosis, thrombocytopenia, qualitative platelet dysfunction, suppressed platelet aggregation, increased vascularity secondary to the release of nitric oxide from endothelium, activation of platelet microparticles, deficiency of coagulation factors, hypofibrinogenemia, and fibrinolysis. These patients are also published online October 22, 2019
DOI https://doi.org/ 10.1055/s-0039-1696912 ISSN 2457-0206.
Copyright @2020 Official

Publication of The Simulation

Society (TSS), accredited

by International Society of

Cardiovascular Ultrasound (ISCU).

\section{License terms}

$(\circledast) \Theta \circledast$ 
susceptible to thrombosis and organ infarction. ${ }^{2}$ This hemorrhagic diathesis is further accentuated by hemodilution, platelet dysfunction, and loss of procoagulants during cardiopulmonary bypass (CPB). ${ }^{3,4}$

The blood loss and coagulopathy in these patients is treated by transfusion of packed red blood cells (PRBCs), fresh frozen plasma (FFP), platelets, and cryoprecipitate. However, transfusion of blood components is associated with numerous complications like transfusion-related acute lung injury (TRALI), transfusion-related circulatory overload (TACO), anaphylactic reactions, transmission of infectious diseases, and hemolytic and nonhemolytic transfusion reactions., This may increase the morbidity, duration of ventilatory support, duration of intensive care unit (ICU) stay, postoperative outcome, and hospital cost. Hence, judicious use of these blood components under guidance of blood coagulation testing is warranted.

The hemostasis management after cardiac surgery is usually guided by conventional laboratory tests like platelet count, prothrombin time (PT), activated partial thromboplastin time (aPTT), and serum fibrinogen level. ${ }^{7}$ However, the value of these tests has been questioned due to their poor ability to predict bleeding and prolonged turnaround time ${ }^{8}$.

In the last decade, viscoelastic point-of-care (POC) coagulation devices like rotational thromboelastometer (ROTEM, TEM International GmbH, Munich, Germany) and Sonoclot (Sienco Inc., Arvada, Colorado) have been increasingly used in major surgeries for timely assessment and management of coagulopathies. The hemostatic therapy based on POC testing has been shown to reduce transfusion of blood products and provide significant benefits with respect to postoperative outcomes in adult cardiac surgery patients. ${ }^{9-16}$

This study was performed to evaluate the efficacy of POC (ROTEM and Sonoclot) guided hemostatic management in cyanotic congenital cardiac surgery patients.

\section{Material and Methods}

\section{Trial Design}

This prospective, single-centered, randomized control trial was conducted after approval of the institutional ethics committee.

\section{Participants}

The participants were patients older than 10 years, scheduled for elective cardiac surgery on CPB for cyanotic congenital heart surgery, who after heparin reversal following $\mathrm{CPB}$, fulfilled at least one of the two inclusion criteria: (1) diffuse bleeding from capillary bed at wound surfaces requiring hemostatic therapy as assessed by the anesthesiologist and surgeon by inspecting the operative field and/or (2) postoperative (during the first 24 postoperative hours) blood loss exceeding $2 \mathrm{~mL} / \mathrm{kg} / \mathrm{hr}$.

After enrolment in the study, patients were randomly assigned to the conventional group or POC group according to random number table.

Patients undergoing redo surgery were excluded.

\section{Primary Outcomes}

The primary outcomes were amount of PRBC, FFP, platelets, and cryoprecipitate transfused.

\section{Secondary Outcomes}

The secondary put comes were chest drain output at 6 , 12 , and 24 hours; duration of ventilatory support; duration of ICU stay; duration of hospital stay; and number of re-explorations.

\section{Anesthesia and Cardiopulmonary Bypass Management}

Routine pre-anesthetic examination was performed one day prior to surgery. The procedure was explained to the parent and written informed consent was obtained. Anesthetic, surgical, and cardiopulmonary bypass management was standardized in all patients. Anesthesia was induced with ketamine (1-2 mg/kg), fentanyl (2-3 $\mu \mathrm{g} / \mathrm{kg})$, and rocuronium bromide $(0.8-1 \mathrm{mg} / \mathrm{kg})$. Maintenance of anesthesia was achieved with sevoflurane $(0.5-1 \%)$ in oxygen-air mixture with intermittent doses of fentanyl, midazolam, and vecuronium. All the patients were monitored with five lead $\mathrm{ECG}, \mathrm{SpO}_{2}$, invasive blood pressure, central venous pressure, temperature, and urine output. Transesophageal echocardiography was performed in patients weighing more than $5 \mathrm{~kg}$. Baseline activated clotting time (ACT) was noted prior to systemic heparinization. Patients were heparinized with $4 \mathrm{mg} / \mathrm{kg}$ unfractionated heparin to achieve a target ACT of more than 480 seconds.

A membrane oxygenator was used for all patients during CPB. The CPB circuit was primed with lactated ringer solution $20 \mathrm{~mL} / \mathrm{kg}$, sodium bicarbonate (7.5\%) $1 \mathrm{~mL} / \mathrm{kg}$, mannitol (20\%) $0.5 \mathrm{~g} / \mathrm{kg}$ and $100 \mathrm{U} / \mathrm{kg}$ of unfractionated heparin. PRBCs were added to pump volume during CPB, to maintain a target hematocrit of $>30 \%$. Myocardial preservation was achieved by administering del Nido cardioplegia and maintaining mild hypothermia $\left(32^{\circ} \mathrm{C}\right)$. Blood gas analyses and ACT were performed intraoperatively at half hourly intervals. Systemic pump flows were maintained between 120 and $200 \mathrm{~mL} / \mathrm{kg} / \mathrm{min}$. Magnesium sulfate $50 \mathrm{mg} / \mathrm{kg}$ was administered on CPB at aortic cross clamp removal. Conventional hemofiltration was done in all the patients.

At the end of the surgery, all the patients were rewarmed to $36^{\circ} \mathrm{C}$ and were weaned from $\mathrm{CPB}$. All patients were reversed with protamine $1.3 \mathrm{mg} / \mathrm{kg}$ of heparin slowly over 10 minutes. Blood and blood components were administered according to the results of conventional or POC tests. All patients were transferred to ICU for elective postoperative ventilation.

\section{Hematologic Analyses}

\section{Conventional Coagulation tests}

Laboratory coagulation testing included platelet count, hemoglobin concentration, serum fibrinogen concentration, international normalized ratio and activated partial thromboplastin time and were performed at the hospital laboratory using the fully automated analyzers. Intraoperatively, repetitive blood gas analyses and ACT analyses were performed. Conventional coagulation tests (platelet count, fibrinogen concentration, international normalize ratio, and activated 
partial thromboplastin time) were performed in both groups at fixed time points (preoperatively, at admission to the ICU, and 24 hour after admission to ICU) to enable a comparison of both groups at these time points.

\section{POC Testing}

Thromboelastometry was performed at the bedside using the ROTEM Delta device (ROTEM, Tem International GmbH, Munich, Germany). Platelet function was assessed using Sonoclot (Sienco Inc., Arvada, Colorado). The POC algorithms were based on five tests: the EXTEM and INTEM tests, which reflect the extrinsic or intrinsic initiation of coagulation; the FIBTEM test (platelet-inhibited extrinsic activation), which reflects the contribution of fibrin polymerization to clot firmness; the HEPTEM test (heparinase-modified intrinsic activation), which identifies potential heparin effects compared with the INTEM test; and the sonoclot platelet function. Clotting time [CT (s)] in the EXTEM, INTEM, and HEPTEM tests as well as the maximum clot firmness $(\mathrm{mm})$ and the amplitude of clot firmness 10 minute after CT [A10 ( $\mathrm{mm})$ ] for each test, including FIBTEM, were recorded. POC tests were performed solely in the POC group after randomization to this study group, after therapeutic interventions, and in case of ongoing bleeding after therapeutic interventions.

POC tests were performed at the bedside by the attending physician whereas conventional laboratory analyses were done in the central laboratory by a technician.

\section{Transfusion of PRBC and Blood Components}

PRBC and blood components were administered if there was diffuse bleeding from capillary bed at wound surfaces requiring hemostatic therapy as assessed by the anesthesiologist and surgeon by inspecting the operative field and/or intraoperative or postoperative (during the first 24 postoperative hours) blood loss exceeding $2 \mathrm{~mL} / \mathrm{kg} / \mathrm{hr}$. Hemostatic therapy was administered according to the conventional and POC algorithms only after correcting hypothermia, calcium concentration, and adequate protamine reversal. Additional testing was done as required. Reduction in chest drain output $<2 \mathrm{~mL} / \mathrm{kg} / \mathrm{hr}$ marked the end point of therapy. The POC and conventional algorithms were based on previous studies done in pediatric patients undergoing cardiac surgery ${ }^{17,18}$ (-Figs. 1 and 2 ).

\section{PRBC Transfusion Protocol}

Packed erythrocytes were transfused to maintain a hemoglobin concentration above $10 \mathrm{~g} / \mathrm{dL}$ during CPB and $12 \mathrm{~g} / \mathrm{dL}$ after CPB. Further indications for the transfusion of packed erythrocytes were (1) central venous oxygen saturation $\left(\mathrm{ScvO}_{2}\right)$ less than $60 \%$ not due to low cardiac output and (2) individual bleeding dynamics strongly indicating the need for erythrocyte transfusion.

\section{FFP Transfusion Protocol}

In the conventional group, FFP were given if a PTT was more than 50 second or international normalized ratio (INR) was more than 1.4 in the dose of $10 \mathrm{~mL} / \mathrm{kg}$. In the POC group, FFP were given if CT EXTEM > 80 / CT HEPTEM > 240 in a dose of $10 \mathrm{~mL} / \mathrm{kg}$.

\section{Platelet Transfusion Protocol}

In the conventional group, platelets were transfused in a dose of 1 unit/10 kg (each unit is of $50 \mathrm{~mL}$ ) if platelet count was less than $1,00,000 / \mu \mathrm{L}$. In the POC group, platelets were transfused if A10 EXTEM $<40 \mathrm{~mm}$, A10 FIBTEM > 7, and sonoclot platelet function $(\mathrm{PF})<1.5$.

\section{Cryoprecipitate Transfusion Protocol}

In the conventional group, cryoprecipitate was administered in a dose of $1 \mathrm{unit} / 10 \mathrm{Kg}$ (each unit contains $25 \mathrm{~mL}$ ) if serum fibrinogen levels were $<150 \mathrm{mg} / \mathrm{dL}$. In the POC group, cryoprecipitate was administered if A10 EXTEM was $<40 \mathrm{~mm}$, sonoclot PF $>1.5$, and A10 FIBTEM $<7$.

\section{Assessment of Hemostasis and Hemostatic Therapy}

The conventional tests, that is, aPTT, INR, and serum fibrinogen levels were used to assess the hemostasis and the effect hemostatic therapy in the both the groups. The tests were performed at the ICU admission, 12 hours after ICU admission, and 24 hours after ICU admission.

\section{Sample Size Calculation and Statistics}

In a previous study, it was shown that performing algorithm-based hemostatic therapy was associated with a $28 \%$ decrease in the mean number of transfused PRBC. We anticipated at least $20 \%$ reduction in the amount PRBC transfused. Considering 5\% $\alpha$ error and $80 \%$ power, the sample size was 85 cases each group. SPSS version 24 was used for statistical analysis. Pearson chi-square test was used for categorical data. As the data was normally distributed, unpaired $t$-test was used to describe the differences between the two groups for parametric data. ${ }^{19}$

\section{Results}

A total of 170 patients were randomized into two groups, that is, conventional and POC groups. There were no losses or exclusions following randomization.

There were no group differences regarding the demographic, clinical, and laboratory characteristics (-Table 1).

Routine laboratory analyses were obtained at the start of surgery and until 24 hours of ICU admission. The baseline values show slightly reduced fibrinogen concentration, platelet count, increased aPTT, ACT, and INR. There were no differences between the two groups in terms of the routine laboratory tests at different time intervals (-Table 2).

The primary outcomes were the amount of blood and blood products consumed in each group. In the POC group, $25.42 \mathrm{~mL} / \mathrm{kg}$ PRBC were used while in conventional group, $30.87 \mathrm{~mL} / \mathrm{kg}$ PRBC were used $(p<0.001)$. In the POC group, $16.45 \mathrm{~mL} / \mathrm{kg}$ of FFP was used while in conventional roup, $20.96 \mathrm{~mL} / \mathrm{kg}$ of FFP was used $(p<0.001)$. In thePOC group, $10.01 \mathrm{~mL} / \mathrm{kg}$ platelet was used, while in the conventional group, $13.9 \mathrm{~mL} / \mathrm{kg}$ of platelet was used $(p<0.001)$. In the POC 

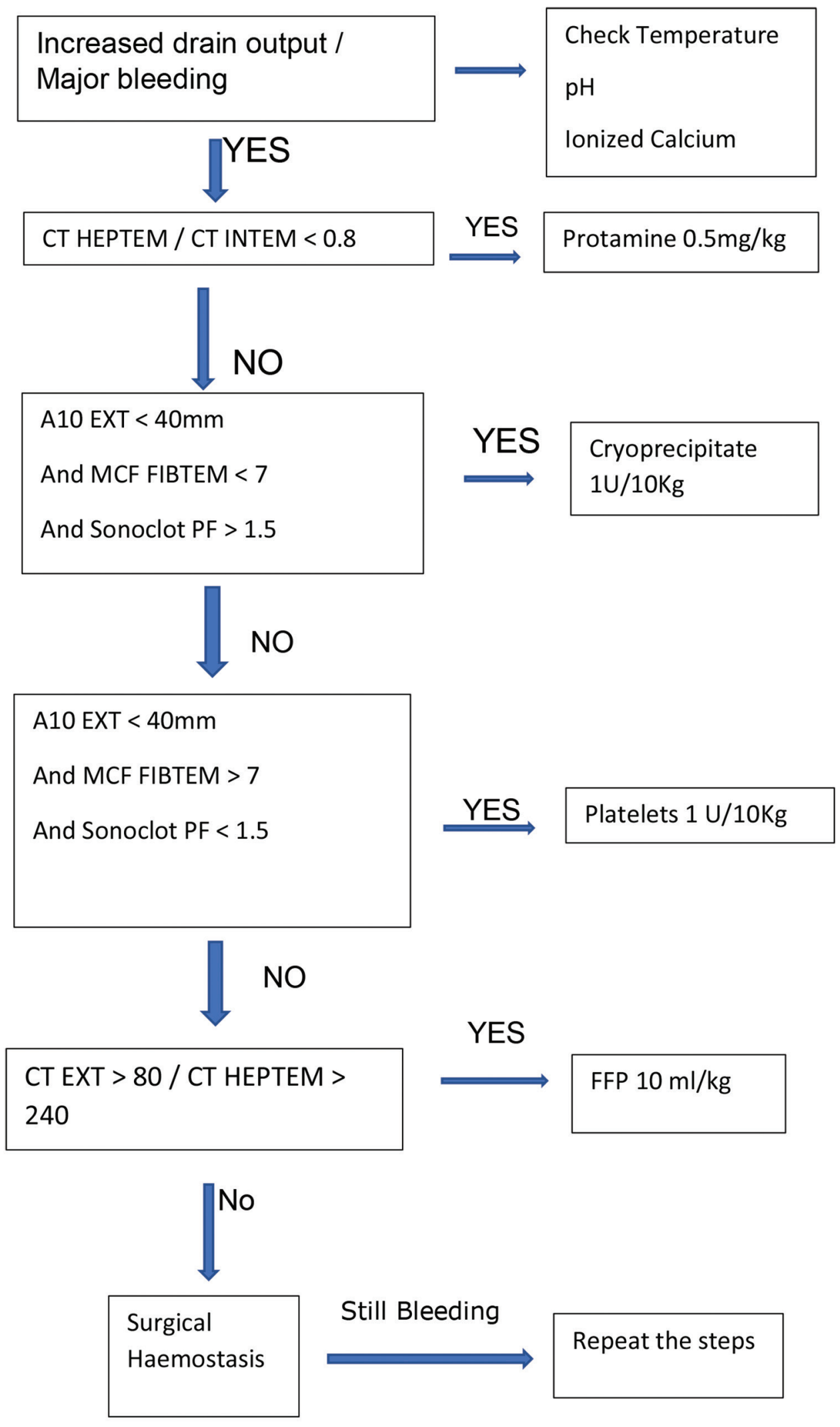

Fig. 1 Point of care algorithm. 


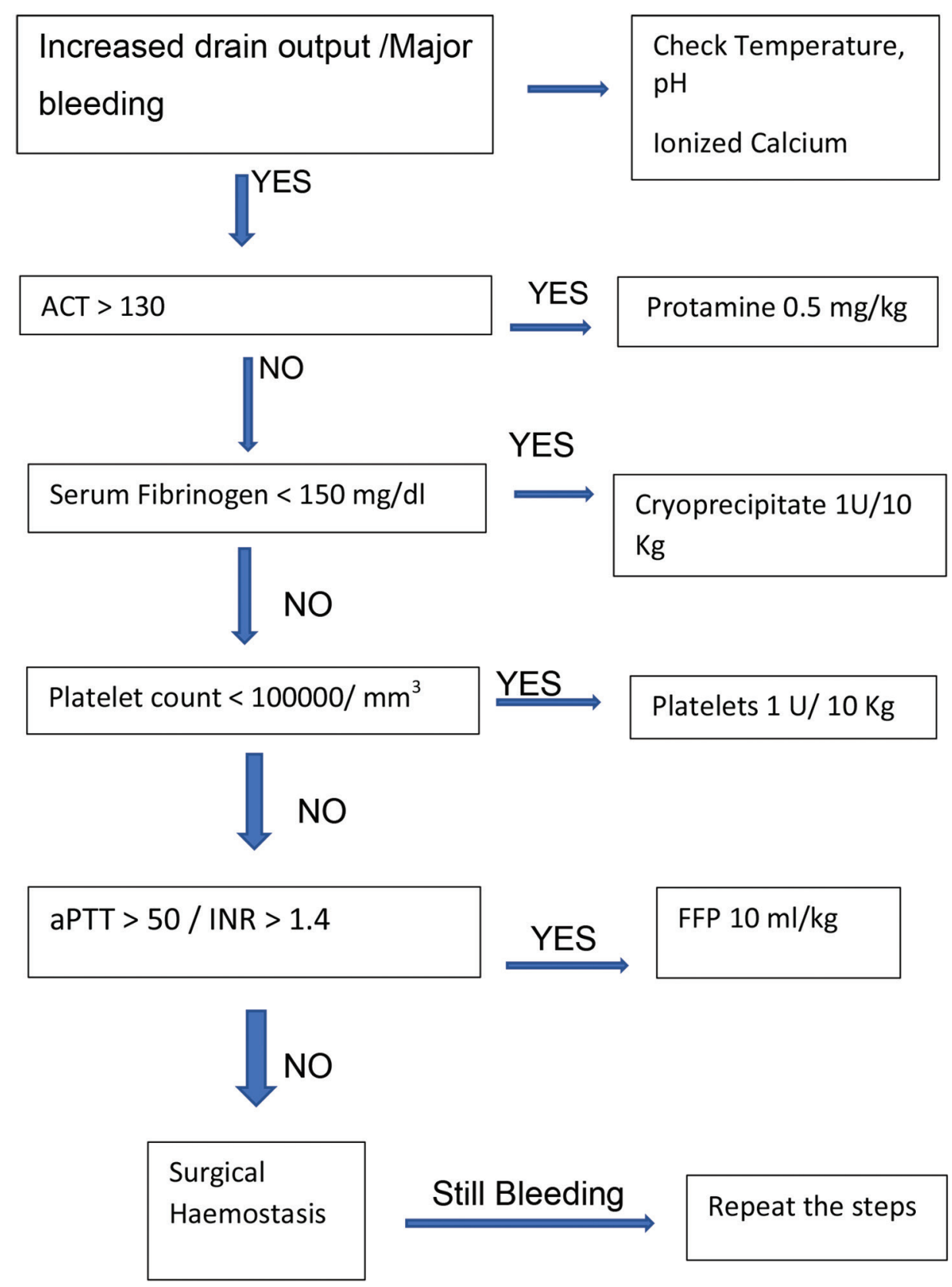

Fig. 2 Conventional algorithm.

group, $7.46 \mathrm{~mL} / \mathrm{kg}$ of cryoprecipitate was used as compared with $11.20 \mathrm{~mL} / \mathrm{kg}$ in conventional group $(p<0.001)$ ( - Table 3 and - Fig. 3).

The secondary outcomes were chest drain output (-Table 4 and -Fig. 4), number of re-explorations, duration of ventilatory support, ICU, and hospital stay (-Table 4 and - Fig. 5). There was no significant difference between the two groups as far as chest drain output and number of re-explorations was considered. In the POC group the mean chest drain output after 24 hours was $8.88 \mathrm{~mL} / \mathrm{kg}$ while in the conventional group, it was $9.54 \mathrm{~mL} / \mathrm{kg}(p=0.234)$. In the POC group, mean duration of ventilatory support was 41.75 hours as compared with 49.58 hours in the conventional group ( $p=0.008$ ). In the POC group, mean duration of ICU stay was 59.67 hours as compared with 76.54 hours in the conventional group $(p<0.001)$. In the POC group, the mean duration of hospital stay was 6.44 days as compared with 7.11 days in the conventional group $(p=0.019)$.

\section{Discussion}

The study compared the two algorithms, one based on POC devices and other based on the standard tests in treating coagulopathy after weaning from the cardiopulmonary bypass in pediatric cyanotic cardiac surgery patients. The results showed that POC-guided hemostatic therapy leads 
Table 1 Comparison of demographic characteristics

\begin{tabular}{|l|l|l|l|}
\hline Parameters & Point of care $(\boldsymbol{n}=85)$ & Conventional $(\boldsymbol{n}=85)$ & $p$-Value \\
\hline Age $($ years) & $4.33 \pm 2.19$ & $4.36 \pm 1.82$ & 0.909 \\
\hline Male sex $(\%)^{\mathrm{a}}$ & $57.6 \%$ & $49.4 \%$ & 0.356 \\
\hline${\text { Weight }(\mathrm{kg})^{\mathrm{a}}}^{\mathrm{a}}$ & $12.27 \pm 4.97$ & $12.55 \pm 4.55$ & 0.347 \\
\hline Height $(\mathrm{cm})^{\mathrm{a}}$ & $103.99 \pm 14.45$ & $96.73 \pm 20.67$ & 0.566 \\
\hline${\text { CPB time }(\mathrm{min})^{\mathrm{a}}}^{\mathrm{a}}$ & $129.09 \pm 28.89$ & $130.15 \pm 28.17$ & 0.809 \\
\hline${\text { Clamp time }(\mathrm{min})^{\mathrm{a}}}^{\text {Procedure }}$ & $90.55 \pm 20$ & $88.60 \pm 20.19$ & 0.527 \\
\hline ASO $(\%)^{\mathrm{b}}$ & & & \\
\hline Glenn Shunt $(\%)^{\mathrm{b}}$ & 4.1 & 4.7 & 0.849 \\
\hline Fontan $(\%)^{\mathrm{b}}$ & 3.8 & 3.5 & 0.917 \\
\hline ICR $(\%)^{\mathrm{b}}$ & 17.6 & 17.6 & 1 \\
\hline ICR+LPA plasty $(\%)^{\mathrm{b}}$ & 66.1 & 67.1 & 0.890 \\
\hline ICR+RPA plasty $(\%)^{\mathrm{b}}$ & 5.7 & 4.7 & 0.769 \\
\hline
\end{tabular}

Abbreviations: ASO, arterial switch operation; CPB, cardiopulmonary bypass; ICR, intracardiac repair for tetralogy of Fallot; LPA, left pulmonary artery; RPA, right pulmonary artery.

anpaired t-test used to test the statistical significance.

bPearson chi-square test used to test the statistical significance.

Note: Values were expressed as mean \pm standard deviation.

Table 2 Laboratory analyses at various time intervals

\begin{tabular}{|c|c|c|c|}
\hline Parameters & Point of care $(n=85)$ & Conventional $(n=85)$ & $p$-Value \\
\hline HB baseline (g/dL) & $16.25 \pm 2.40$ & $16.34 \pm 2.31$ & 0.795 \\
\hline HB at ICU admission & $10.25 \pm 2.42$ & $9.99 \pm 2.28$ & 0.472 \\
\hline HB $24 \mathrm{hr}$ & $12.25 \pm 2.40$ & $13.22 \pm 2.26$ & 0.937 \\
\hline ACT baseline (s) & $151.35 \pm 14.43$ & $150.27 \pm 14.53$ & 0.627 \\
\hline ACT ICU admission & $131.95 \pm 14.12$ & $129.54 \pm 17.26$ & 0.320 \\
\hline Fibrinogen baseline (mg/dL) & $176.18 \pm 87.76$ & $179.25 \pm 89.10$ & 0.821 \\
\hline Fibrinogen at ICU admission & $146.24 \pm 72.83$ & $153.59 \pm 77.31$ & 0.524 \\
\hline Fibrinogen at $24 \mathrm{hr}$ & $187.21 \pm 93.22$ & $196.72 \pm 98.47$ & 0.519 \\
\hline APTT baseline (s) & $40.33 \pm 22.86$ & $37.01 \pm 8.06$ & 0.209 \\
\hline APTT at ICU admission & $46.55 \pm 26.37$ & $43.20 \pm 9.30$ & 0.270 \\
\hline APTT $24 \mathrm{hr}$ & $32.56 \pm 18.45$ & $33.67 \pm 7.19$ & 0.607 \\
\hline INR baseline & $1.33 \pm 0.47$ & $1.32 \pm 0.47$ & 0.871 \\
\hline INR at ICU admission & $1.60 \pm 0.49$ & $1.56 \pm 0.52$ & 0.651 \\
\hline INR $24 \mathrm{hr}$ & $1.03 \pm 0.15$ & $1.0 \pm 0.19$ & 0.310 \\
\hline $\mathrm{pH}$ at ICU admission & $7.38 \pm 0.17$ & $7.37 \pm 0.18$ & 0.096 \\
\hline Lactate baseline (mmol/L) & $2.39 \pm 3.68$ & $2.19 \pm 3.24$ & 0.707 \\
\hline Lactate at ICU admission & $1.99 \pm 2.96$ & $1.84 \pm 2.67$ & 0.724 \\
\hline Lactate at $24 \mathrm{hr}$ & $1.55 \pm 2.41$ & $1.54 \pm 2.70$ & 0.697 \\
\hline Platelet count baseline $(\mu \mathrm{L})$ & $177.19 \pm 78.62$ & $188.79 \pm 76.94$ & 0.332 \\
\hline Platelet count at ICU admission & $152.19 \pm 78.62$ & $171.73 \pm 76.83$ & 0.103 \\
\hline Platelet count after $24 \mathrm{hr}$ in ICU & $182.19 \pm 76.62$ & $193.69 \pm 77.35$ & 0.338 \\
\hline $\begin{array}{l}\text { Temperature at ICU admission } \\
\text { (degree Celsius) }\end{array}$ & $36.26 \pm 4.44$ & $35.67 \pm 4.43$ & 0.911 \\
\hline $\mathrm{PaO}_{2} / \mathrm{FiO}_{2}$ at $6 \mathrm{hr}$ & $319.64 \pm 85.64$ & $355.85 \pm 76.34$ & 0.004 \\
\hline $\mathrm{PaO}_{2} / \mathrm{FiO}_{2}$ at $12 \mathrm{hr}$ & $423.61 \pm 93.884$ & $358.58 \pm 96.90$ & 0.001 \\
\hline
\end{tabular}

Abbreviations: ACT, activated clotting time; $\mathrm{FiO}_{2}$, fraction of inspired oxygen; $\mathrm{HB}$, hemoglobin; $\mathrm{PaO}_{2}$, partial pressure of oxygen.

Note: Unpaired $t$-test used to test the statistical significance. Values expressed as mean \pm standard deviation. 
Table 3 Primary outcomes

\begin{tabular}{|l|l|l|l|}
\hline & Point of care & Conventional & $p$-Value \\
\hline PRBC used $(\mathrm{mL} / \mathrm{kg})(\mathrm{n})$ & $25.42 \pm 4.227(85)$ & $30.87 \pm 1.211(85)$ & $<0.001$ \\
\hline FFP used $(\mathrm{mL} / \mathrm{kg})(\mathrm{n})$ & $16.45 \pm 3.797(81)$ & $20.96 \pm 5.161(82)$ & $<0.001$ \\
\hline Platelets used $(\mathrm{mL} / \mathrm{kg})(\mathrm{n})$ & $10.01 \pm 2.541(69)$ & $13.90 \pm 2.462(80)$ & $<0.001$ \\
\hline Cryoprecipitate Used $(\mathrm{mL} / \mathrm{kg})(\mathrm{n})$ & $7.46 \pm 1.866(71)$ & $11.20 \pm 2.512(78)$ & $<0.001$ \\
\hline
\end{tabular}

Abbreviations: FFP, fresh frozen plasma; $n$, number of patients who received therapy; PRBC, packed red blood cells.

Note: Unpaired $t$-test used to test the statistical significance. Values expressed as mean \pm standard deviation.

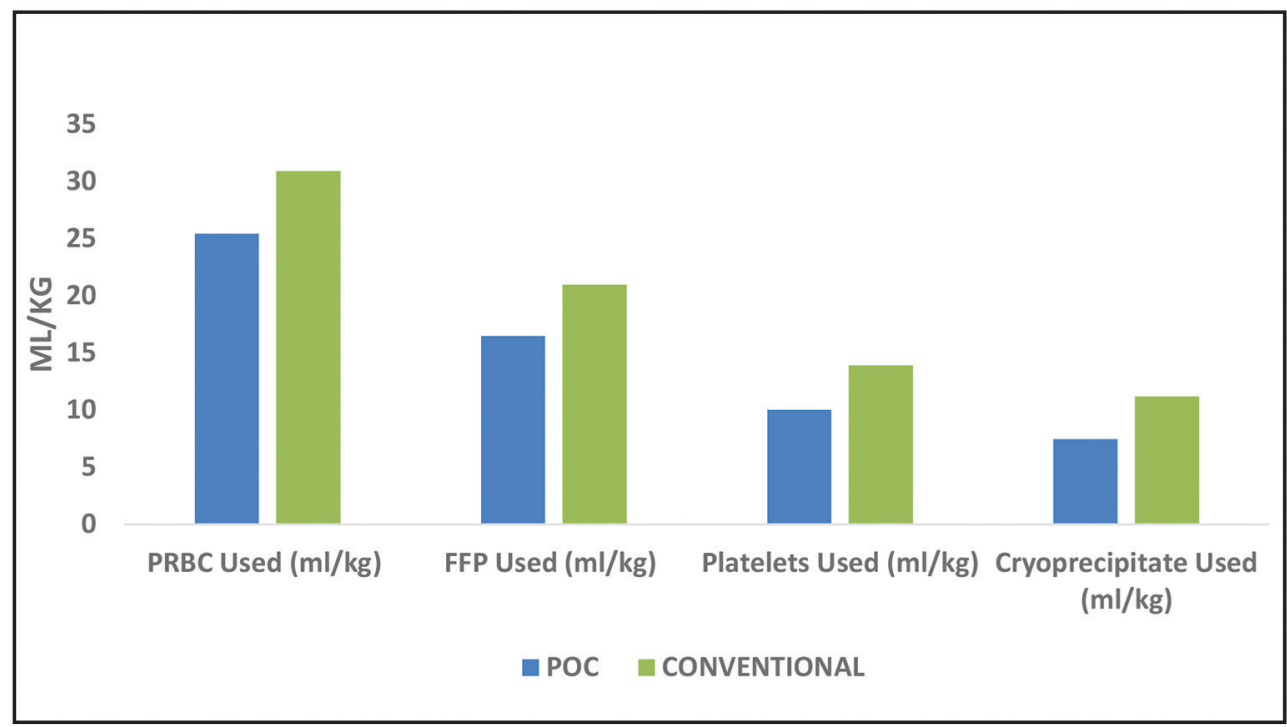

Fig. 3 Comparison of blood and blood products.

Table 4 Secondary outcomes

\begin{tabular}{|c|c|c|c|}
\hline & Point of care $(n=85)$ & Conventional $(n=85)$ & $p$-Value \\
\hline Chest drain $6 \mathrm{hr}(\mathrm{mL} / \mathrm{kg})^{\mathrm{a}}$ & $5.46 \pm 2.079$ & $6.02 \pm 2.32$ & 0.097 \\
\hline Chest drain $12 \mathrm{hr}(\mathrm{mL} / \mathrm{kg})^{\mathrm{a}}$ & $7.42 \pm 2.714$ & $8.89 \pm 3.41$ & 0.002 \\
\hline Chest drain $24 \mathrm{hr}(\mathrm{mL} / \mathrm{kg})^{\mathrm{a}}$ & $8.88 \pm 3.396$ & $9.54 \pm 3.79$ & 0.234 \\
\hline Re-explorations ${ }^{\mathrm{b}}$ & $1.2 \%$ & $1.6 \%$ & 0.824 \\
\hline Ventilatory support (hr) ${ }^{\mathrm{a}}$ & $41.75 \pm 16.744$ & $49.58 \pm 20.95$ & 0.008 \\
\hline ICU duration (hr) ${ }^{\mathrm{a}}$ & $59.67 \pm 25.330$ & $76.54 \pm 22.12$ & $<0.001$ \\
\hline Hospital stay (days) ${ }^{\mathrm{a}}$ & $6.44 \pm 1.686$ & $7.11 \pm 1.99$ & 0.019 \\
\hline
\end{tabular}

Abbreviation: ICU, intensive care unit.

anpaired t-test used to test the statistical significance.

bPearson chi-square test used to test the statistical significance.

Note: Values expressed as mean \pm standard deviation.

to reduced perioperative exposure to blood products and improved clinical outcome. Also, POC tests had reduced turnaround time than the conventional tests. This finding is similar to the finding in other studies. There was no difference between the two groups in terms of the temperature, $\mathrm{pH}$, and serum lactate levels.

The amount of PRBC administered was significantly higher in the conventional group as compared with the POC group (30.87 mL/kg vs. $25.42 \mathrm{~mL} / \mathrm{kg} p<0.001$ ). This is consistent with the previous studies in which the amount of PRBC transfused was significantly less in the POC group. ${ }^{18,20}$ This difference may be due to the fact that the conventional tests had longer turnaround time which led to higher amount of PRBC administration.

The amount of FFP, platelets, and cryoprecipitate administered was significantly higher in the conventional group $(p<0.001)$, which is consistent with other pediatric and adult studies. ${ }^{14,17,18,21}$ This finding is particularly important in pediatric surgeries as the amount of blood and blood products transfused can cause transfusion-associated acute lung injury (TRALI) and anaphylactic reactions, and adversely affect the 


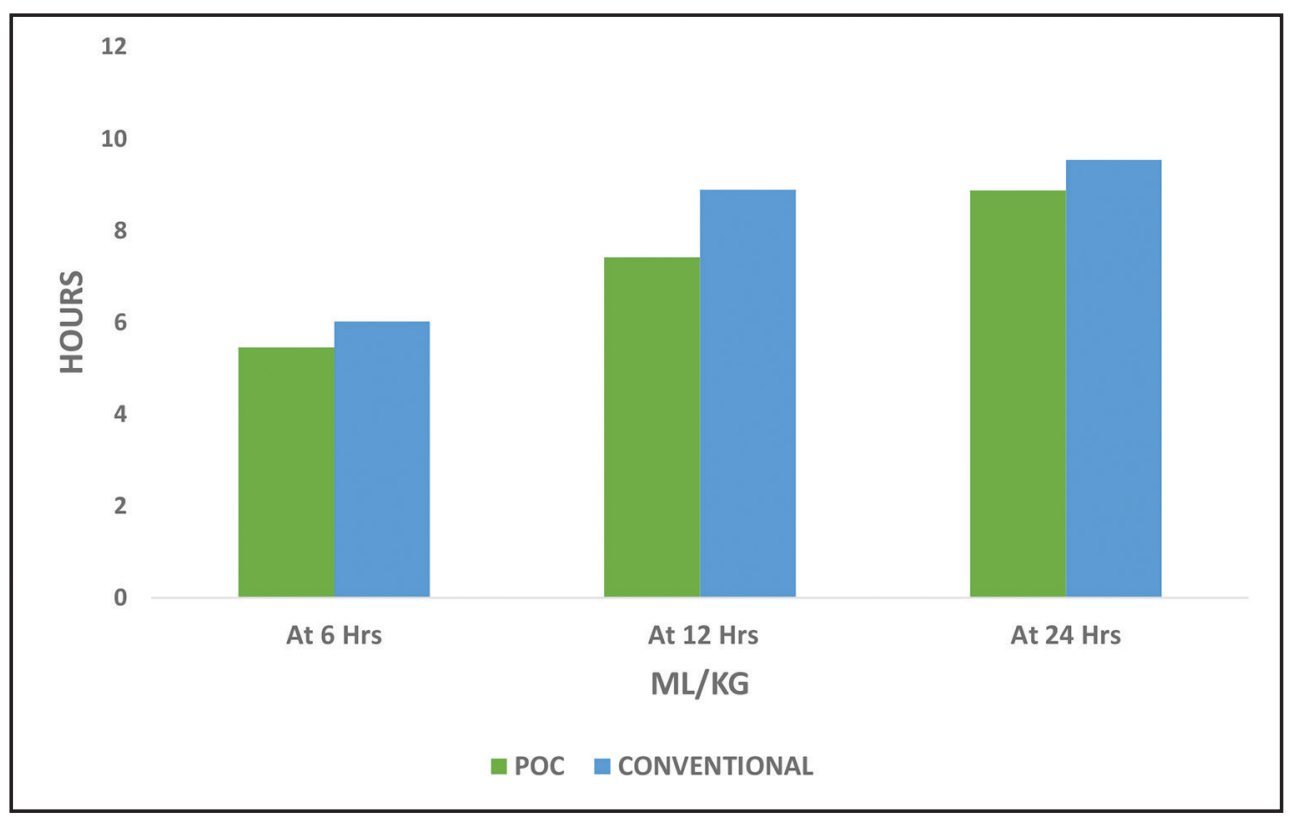

Fig. 4 Comparison of chest drain output.

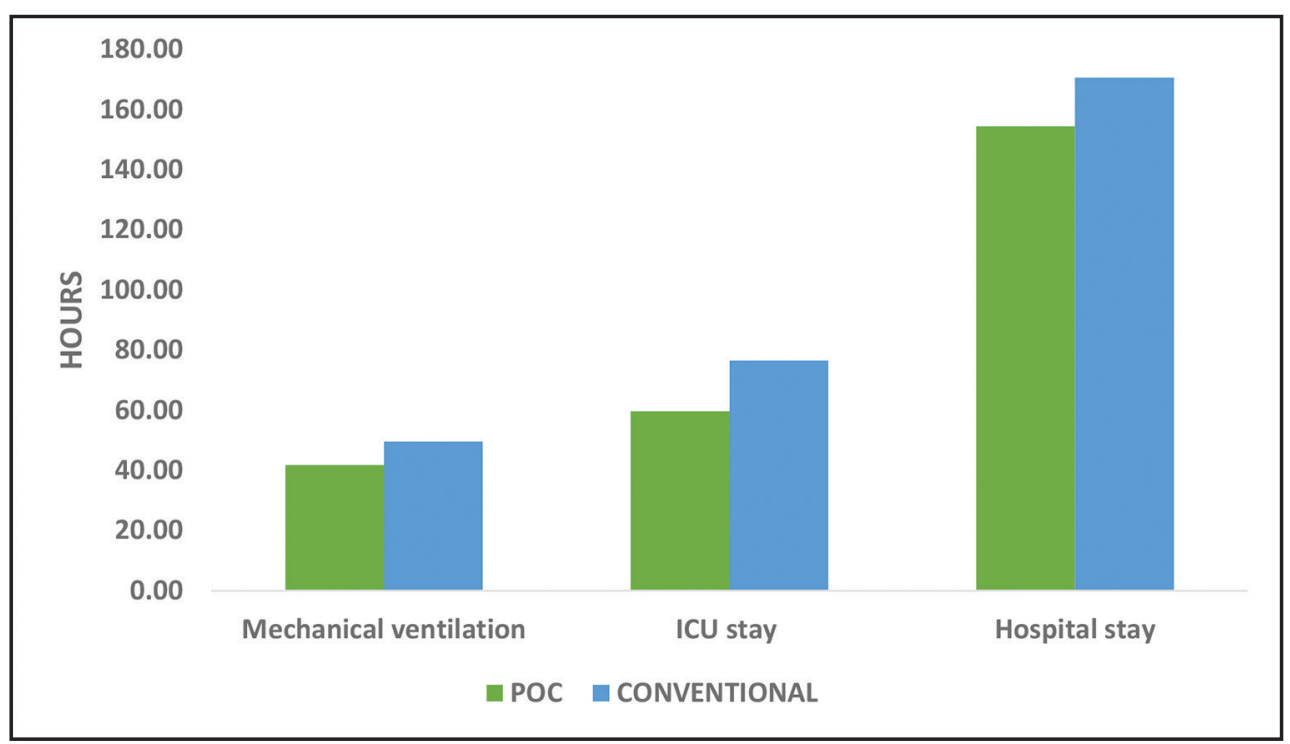

Fig. 5 Comparison of secondary outcomes.

patient outcome..$^{22,23}$ In addition, the tetralogy patients who receive transannular patch for relief of valvular stenosis, this significant increase in circulatory blood volume in background of concomitant pulmonary valvular regurgitation can precipitate

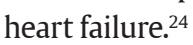

There was no significant difference in the postoperative chest drain output at 6,12 , and 24 hours after the surgery ( $p=0.097,0.141,0.234$, respectively). This finding is contradictory to the similar study done by Nakayama et al, in which they found reduced 24-hour chest drain output in the POC group (13 mL/kg vs. $22 \mathrm{~mL} / \mathrm{kg}, p=0.002$ ). This may be due to excess amount of blood products transfused in the conventional group. Though the chest drain output was not significantly different in the two groups, it came at a cost of significantly more transfusion emphasizing the ability of POC tests in avoiding the over transfusion and associated adverse effects.

The mean duration of mechanical ventilation was significantly more in the conventional group as compared with the POC group (41.75 hours vs. 49.58 hours $p=0.008$ ). The finding is justified by the significantly reduced $\mathrm{PaO}_{2} / \mathrm{FiO}_{2}$ in the conventional group at 6 and 12 hours. This may have occurred due to the significantly more amount of blood products administered in the conventional group causing circulatory overload and TRALI. Also, the duration of ICU stay and hospital stay was increased in the conventional group. These findings are similar to the findings of the other studies. ${ }^{14,25,26}$ 


\section{Limitations}

This was a single-center study. So interinstitutional variations couldn't be considered. The POC algorithm used in the study is a simple and two point of care devices were used due to non-availability of platelet aggregometry. Availability of recombinant factor VII, prothrombin, and fibrinogen concentrates could have caused a further reduction in the amount of transfusion. The study population was small to draw conclusion about mortality and long-term morbidity.

\section{Conclusion}

Implementation of POC-based algorithm-guided hemostatic therapy reduced the exposure to blood and blood component therapy and was associated with reduced ICU and hospital stay in pediatric congenital cyanotic surgical patients. FIBTEM is a sensitive marker with EXTEM for hemostatis.

\section{Conflict of Interests}

None.

\section{Acknowledgment}

This investigation was funded by research grant under the JCCC-TSS banner. The research aid was given for equipment and reagents by Vijyoti Management, New Delhi.

\section{References}

1 Ghasemi A, Horri M, Salahshour Y, et al. Coagulation abnormalities in pediatric patients with congenital heart disease: A Literature Review. Int J Pediatr 2014;2:141-143

2 Horigome H, Hiramatsu Y, Shigeta O, Nagasawa T, Matsui A. Overproduction of platelet microparticles in cyanotic congenital heart disease with polycythemia. J Am Coll Cardiol 2002;39(6):1072-1077

3 Despotis GJ, Gravlee G, Filos K, Levy J. Anticoagulation monitoring during cardiac surgery: a review of current and emerging techniques. Anesthesiology 1999;91(4):1122-1151

4 Bolliger D, Szlam F, Levy JH, Molinaro RJ, Tanaka KA. Haemodilution-induced profibrinolytic state is mitigated by fresh-frozen plasma: implications for early haemostatic intervention in massive haemorrhage. Br J Anaesth 2010;104(3):318-325

5 Kiefel V. Reactions induced by platelet transfusions. Transfus Med Hemother 2008;35(5):354-358 10.1159/000151350

6 Pandey S, Vyas GN. Adverse effects of plasma transfusion. Transfusion 2012;52(Suppl 1):65S-79S

7 Nuttall GA, Oliver WC, Ereth MH, Santrach PJ. Coagulation tests predict bleeding after cardiopulmonary bypass. J Cardiothorac Vasc Anesth 1997;11(7):815-823

8 SegalJB, Dzik WH; Transfusion Medicine/Hemostasis Clinical Trials Network. Paucity of studies to support that abnormal coagulation test results predict bleeding in the setting of invasive procedures: an evidence-based review. Transfusion 2005;45(9):1413-1425

9 Spalding GJ, Hartrumpf M, Sierig T, Oesberg N, Kirschke CG, Albes JM. Cost reduction of perioperative coagulation management in cardiac surgery: value of "bedside" thromboelastography (ROTEM) Eur J Cardiothorac Surg 2007;31(6):1052-1057

10 Coakley M, Reddy K, Mackie I, Mallett S. Transfusion triggers in orthotopic liver transplantation: a comparison of the thromboelastometry analyzer, the thromboelastogram, and conventional coagulation tests. J Cardiothorac Vasc Anesth 2006;20(4):548-553
11 Spiess BD, Gillies BSA, Chandler W, Verrier E. Changes in transfusion therapy and re-exploration rate after institution of a blood management program in cardiac surgical patients. J Cardiothorac Vasc Anesth 1995;9(2):168-173

12 Li C, Zhao Q, Yang K, Jiang L, Yu J. Thromboelastography or rotational thromboelastometry for bleeding management in adults undergoing cardiac surgery: a systematic review with meta-analysis and trial sequential analysis. J Thorac Dis 2019;11(4):1170-1181

13 Ronald A, Dunning J. Can the use of thromboelastography predict and decrease bleeding and blood and blood product requirements in adult patients undergoing cardiac surgery? Interact Cardiovasc Thorac Surg 2005;4(5):456-463

14 Weber CF, Görlinger K, Meininger D, et al. Point-of-care testing: a prospective, randomized clinical trial of efficacy in coagulopathic cardiac surgery patients. Anesthesiology 2012;117(3):531-547

15 Deppe AC, Weber C, Zimmermann J, et al. Point-of-care thromboelastography/thromboelastometry-based coagulation management in cardiac surgery: a meta-analysis of 8332 patients. J Surg Res 2016;203(2):424-433

16 Bhardwaj V, Malhotra P, Hasija S, Chowdury UK, Pangasa N. Coagulopathies in cyanotic cardiac patients: an analysis with three point-of-care testing devices (thromboelastography, rotational thromboelastometry, and sonoclot analyzer) Ann Card Anaesth 2017;20(2):212-218

17 Ak K, Isbir CS, Tetik S, et al. Thromboelastography-based transfusion algorithm reduces blood product use after elective CABG: a prospective randomized study. J Card Surg 2009;24(4):404-410

18 Faraoni D, Willems A, Romlin BS, Belisle S, Van der Linden P. Development of a specific algorithm to guide haemostatic therapy in children undergoing cardiac surgery: a single-centre retrospective study. Eur J Anaesthesiol 2015;32(5):320-329

19 Görlinger K, Dirkmann D, Hanke AA, et al. First-line therapy with coagulation factor concentrates combined with pointof-care coagulation testing is associated with decreased allogeneic blood transfusion in cardiovascular surgery: a retrospective, single-center cohort study. Anesthesiology 2011;115(6):1179-1191

20 Nakayama Y, Nakajima Y, Tanaka KA, et al. Thromboelastometry-guided intraoperative haemostatic management reduces bleeding and red cell transfusion after paediatric cardiac surgery. Br J Anaesth 2015;114(1):91-102

21 Kuiper GJAJM, van Egmond LT, Henskens YMC, et al. Shifts of transfusion demand in cardiac surgery after implementation of rotational thromboelastometry-guided transfusion protocols: analysis of the HEROES-CS (HEmostasis Registry of patiEntS in Cardiac Surgery) observational, prospective open cohort database. J Cardiothorac Vasc Anesth 2019;33(2):307-317

22 Karkouti K, McCluskey SA, Callum J, et al. Evaluation of a novel transfusion algorithm employing point-of-care coagulation assays in cardiac surgery: a retrospective cohort study with interrupted time-series analysis. Anesthesiology 2015;122(3):560-570

23 Redlin M, Kukucka M, Boettcher W, et al. Blood transfusion determines postoperative morbidity in pediatric cardiac surgery applying a comprehensive blood-sparing approach. J Thorac Cardiovasc Surg 2013;146(3):537-542

24 Iyengar A, Scipione CN, Sheth P, et al. Association of complications with blood transfusions in pediatric cardiac surgery patients. Ann Thorac Surg 2013;96(3):910-916

25 Kestelli M, Tulukoglu E, Yurekli II, Gurbuz A. Think twice while inserting a transannular patch. Eur J Cardiothorac Surg 2009;35(6):1112-1113, author reply 1113

26 Pearse BL, Smith I, Faulke D, et al. Protocol guided bleeding management improves cardiac surgery patient outcomes. Vox Sang 2015;109(3):267-279 